\title{
ON THE EFFECTIVENESS OF OSCILLATING WATER COLUMN DEVICES IN REDUCING THE AGITATION IN FRONT OF VERTICAL WALLS HARBOR STRUCTURES
}

\author{
Lorenzo Cappietti ${ }^{1}$ and Irene Simonetti ${ }^{2}$
}

\begin{abstract}
Reducing wave reflection at vertical wall harbor structures is an important goal to ease mooring and maneuvering inside the port area. In this study, numerical simulations have been carried out to assess the effectiveness of Oscillating Water Column devices as anti-reflective systems to be integrated in vertical wall harbor structures. The numerical simulations have been carried out in a Numerical Wave Tank, implemented in the Computational Fluid Dynamics environment OpenFOAM $®$. A methodological approach to separate the reflected and the radiated wave components is presented. The interaction (destructive/constructive interference) between the reflected and the radiated wave field is studied. Furthermore, a preliminary assessment of the effect of basic design parameters of the Oscillating Water Column on the wave field in front of the structure is discussed. A relatively good performance of the device is found, with a minimum reflection coefficient of around $15 \%$, suggesting that the device could efficiently be used to reduce wave agitation in front of vertical wall harbor structures.
\end{abstract}

Keywords: Anti-Reflective System, Oscillating Water Column, Harbor Agitation, Wave Reflection, Wave-Structure Interaction, Radiated Wave Field, Numerical Modelling, OpenFOAM.

\section{INTRODUCTION}

Wave reflection at harbor structures negatively affects the navigability of entrance canals and harbor tranquility. In case of rubble-mound structures this phenomenon is relatively limited if compared to vertical-wall structures. However, for deep water applications, the use of the vertical-wall structures is an almost obliged choice due to economic reasons. Vertical wall structures are also the preferred choice of harbor managers since they permit a better design of berthing structures and offer an effective approach for reducing the space occupied inside the harbor area, i.e. limiting the footprint of the mooring structure. Reducing the wave reflection at vertical wall structures is thus an important measure. To date, several low-reflectivity structures, to cope with this specific problem, have been presented in the literature, as reviewed in Oumeraci, 2010 and Huang et al., 2011.

The effectiveness of slotted vertical perforated-walls has been studied since many years, with the earliest studies being those by Jarlan, 1961, and the most recent ones by Neelamani et al., 2017. Such kind of structure is often adopted as frontal and internal wall of caisson breakwaters embodying one or multiple chambers (Garrido et al. 2010; Gonzalez-Escriva \& Medina, 2012; Ciocan et al., 2017; Lopez et al., 2018). Caissons with internal rubble mound were also proposed with the aim to reduce inner harbor agitation (Teocharis et al., 2011, Altomare \& Gironella, 2014).

In a perforated/slotted breakwater, part of the incident wave energy is reflected at the perforated seaward wall and part of it is transmitted inside the wave absorbing chamber (i.e., the caisson), where it is partially dissipated due to different phenomena, e.g. friction losses, turbulence, vortices and resonance effects. The hydraulic performance of such structures is mainly influenced by the structure porosity and the width of the wave absorbing chamber. If a structure with an internal rubble mound is used, the dissipation of the wave energy is mainly due to the formation of turbulent flows tanking place into the rubble mound voids (Altomare \& Gironella, 2014). These energy dissipation mechanisms are, however, more efficient for shorter waves (Gonzalez-Escriva \& Medina, 2012). Furthermore, all aforementioned kind of structures dissipates the energy without attempting to recover it into usable forms.

The so-called Oscillating Water Column (OWC) concept, commonly studied and developed mainly as a wave energy converter (Falcão \& Henriques, 2016), could also represent a viable alternative, if integrated in vertical wall harbor structures, to absorb the incident energy thus decreasing the inherent reflecting behavior. The device consists in a hollow chamber, open below the water level, where the external incident wave action excites the oscillation of the inner water column, which in turn causes a reciprocating airflow to move towards an air turbine. Studies that investigates the wave energy harvesting performance of such device are widespread (e.g., Morris-Thomas et al., 2007; Lopez et al. 2014; He and Huang, 2016a; Elhanafi et al., 2017; Simonetti et al., 2016 and 2017). On the contrary, studies that investigate its effectiveness as anti-reflection device are quite limited (Liu and Geng, 2012, $\mathrm{He}$ and Huang 2016b). Indeed, the wave field in front of an OWC structure has been scarcely

\footnotetext{
${ }^{1}$ DICEA, Civil and Environmental Engineering Department, University of Florence, Italy - GNRAC, Italian Coastal Research Group.

2 DICEA, Civil and Environmental Engineering Department, University of Florence, Italy.
} 
investigated (Scarpetta et al., 2017). An OWC device could reduce wave reflection since it absorbs part of the incident energy in the form of: i) pneumatic energy of the reciprocating air flow towards an orifice (not necessarily harvested in a more useful form by the power take off system); ii) energy entrapped in the vortex shedding mechanism taking place at the OWC front wall (He \& Huang, 2016a; Elhanafi et al., 2017). Moreover, from the opposite point of view, an oscillating air pressure acting on the OWC internal free surface excites the inner water column, that necessarily radiates waves toward the exterior (Sarmento \& Falcao, 1985; Falnes, 2002).

In summary, the wave field in front of the OWC depends on the interaction (which may be either constructive or destructive) between the incident, the reflected and the radiated waves, assuming that the device is integrated into a bottom standing breakwater and therefore no energy is transmitted underneath or behind it.

This paper aims to contribute to the present knowledge on the effectiveness of an OWC, embodied in quay walls or harbor breakwaters, as a way to reduce wave reflection at vertical wall structures. The objective of this work is to study the wave field in front of the OWC device and its sensitivity to the variation of the design parameters, highlighting the contribution of reflected and radiated waves. The remainder of the paper is structured as follows: the methodology adopted to separate incident, reflected and radiated wave components is described; the implementation of a Numerical Wave Tank (NWT), used to conduct the wave-structure interaction study, and a sensitivity analysis on the basic model parameters are reported; results on the OWC performance, as a function of the main design parameters, are provided and discussed. Finally, concluding remarks and outlook are given.

\section{METHODOLOGY}

This work is conducted by using a NWT, based on Computational Fluid Dynamics (CFD) numerical modeling. The two-phases (water and air), Volume of Fluid (VOF) solver of the OpenFOAM® software package is used (interFoam).

The sensitivity to the variation of the principal design parameters of the OWC structure is investigated, namely: i) frontal wall lip draught, ii) chamber length (along wave propagation direction), iii) pressure drop caused by the streaming of air through the orifice connecting the internal air chamber to the atmosphere. Regular waves of different frequencies are tested.

For each set of design parameters and wave conditions, the NWT is used to examine separately the reflected and the radiated wave components by applying the following methodology (Fig. 1): in the $1^{s t}$ step, a 110 wave periods (T)-long numerical run is performed, with a 20T-long initial wave generation interval. The generated wave train propagates along the NWT, reaches the OWC structure, is reflected and propagates in still water, together with the radiated component, back towards the wave generation boundary.

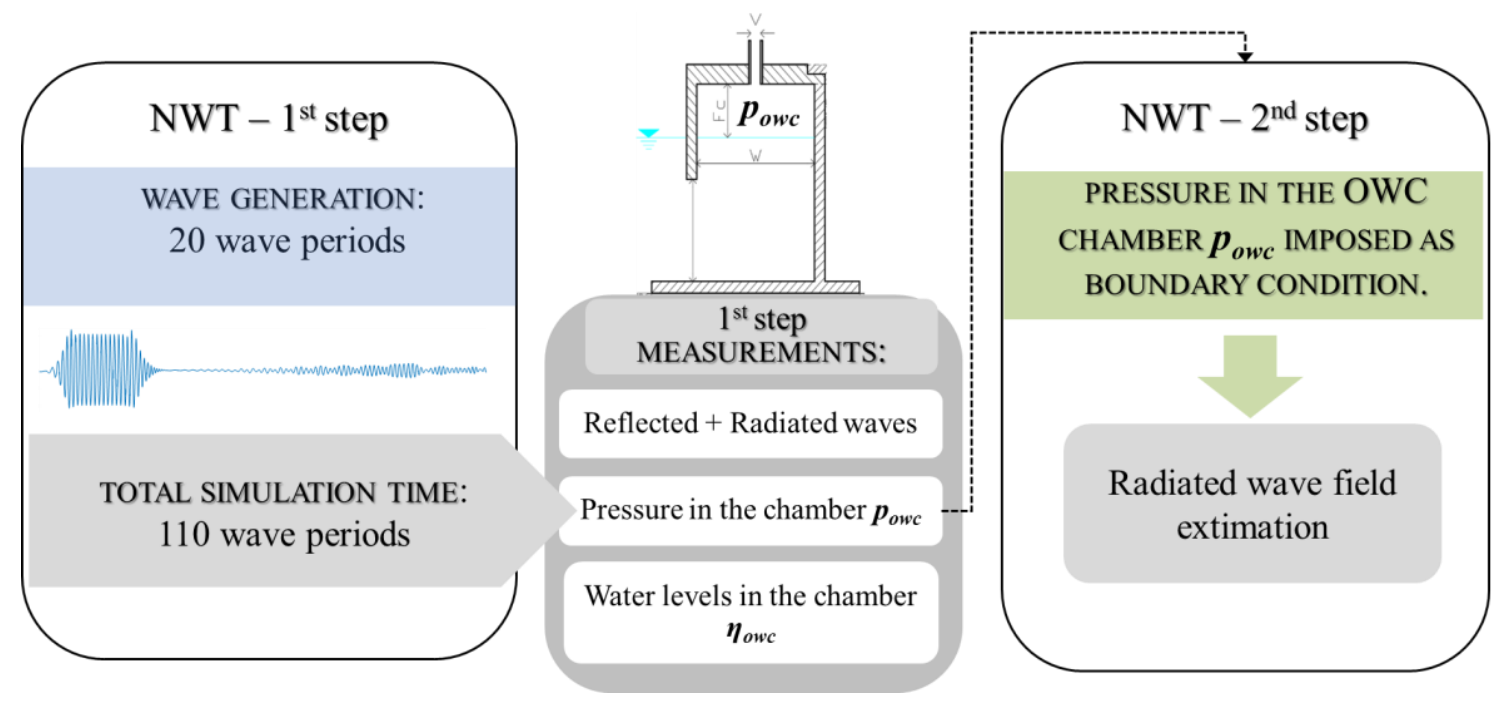

Figure 1. Methodology adopted to separate the reflected and the radiated wave components by simulating the OWC in the Numerical Wave Tank (NWT). 
In this way, it is possible to clearly separate the incident and the reflected plus radiated wave trains in any selected location along the NWT, i.e. to directly measure the reflected plus radiated wave field by means of the zero-crossing analysis, without using spectral methods for separating the incoming and the outgoing wave fields (e.g. Mansard \& Funke, 1980, or Goda \& Suzuki, 1976).

In the simulations of the $1^{\text {st }}$ step, the time series of the air pressure $p_{\text {owc }}$ and the water surface elevation $\eta_{\text {owc }}$ inside the OWC chamber are measured. The recorded time series of $p_{\text {owc }}$ is then imposed as an input for the simulations of the $2^{\text {nd }}$ step: the wave generated in the NWT because of the time-varying pressure inside the OWC chamber is measured (i.e., the radiated wave field is explicitly quantified).

\section{GEOETRIC PARAMETERS OF THE OWC MODEL AND TEST CONDITIONS}

The OWC structure studied in this work consists in a rectangular shaped hollow chamber, conceived to be embedded into a vertical-wall harbor structure (Fig. 2). A water depth of $h=9 \mathrm{~m}$ is considered, with a fixed value of the OWC freeboard $F c=1.5 \mathrm{~m}$, values of the chamber width $W$ varying between 2.5 and $3.5 \mathrm{~m}$ and values of the front wall draught $D$ between 1 and $2 \mathrm{~m}$. The opening on the top of the chamber has an aperture of $V=0.075-0.3 \mathrm{~m}$. Regular wave attacks are considered, with height $H=0.6 \mathrm{~m}$ and periods $T$ ranging between 3 and $5 \mathrm{~s}$. Water depth and wave features have been chosen taking into consideration a relatively low-energy environment that might characterize a location in a harbor area.

Numerical simulations are performed in the NWT considering a 1:15 scale model of the device. Geometry and wave conditions are scaled by using Froude similarity.

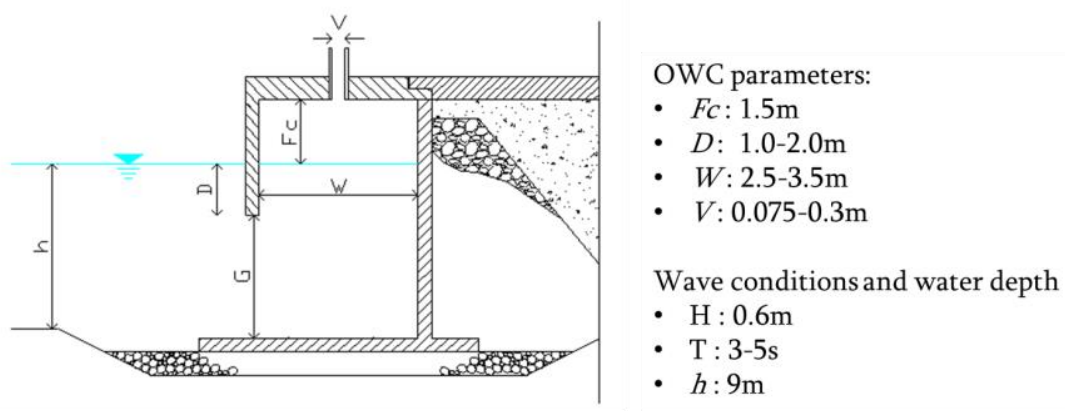

Figure 2. Left: sketch of the OWC integrated into the vertical wall harbor structures; Right: value of the considered design parameters and waves conditions (measurements at full scale).

\section{SET-UP OF THE NUMERICAL WAVE TANK AND SENSITIVITY TESTS}

As aforementioned, the study is carried out by simulating the OWC device in a two-dimensional NWT. The calculations are performed by solving mass conservation (Eq. 1) and the Reynolds Averaged Navier-Stokes (RANS) equations (Eq. 2) for two incompressible phases by using the solver interFoam.

$$
\nabla \cdot U=0
$$

$$
\begin{aligned}
\frac{\partial \rho U}{\partial t}+\nabla \cdot(\rho U \otimes U) & -\nabla \cdot\left(\mu_{e f f} \nabla U\right)= \\
& -\nabla p-g \cdot x \nabla \rho+\nabla U \cdot \nabla \mu_{e f f}+\sigma \kappa \nabla \gamma
\end{aligned}
$$

where $U$ is the fluid velocity vector, $\rho$ is the fluid density, $\mu_{\text {eff }}$ is the efficient dynamic viscosity (defined as $\mu_{e f f}=\mu+\mu_{t}$, with $\mu$ being the molecular dynamic viscosity and $\mu_{t}$ being the turbulent viscosity), $p$ is the pressure, $g$ is the acceleration of gravity vector, $x$ is the position vector, $\sigma$ is the surface tension coefficient, $\kappa$ is the interface curvature and $\gamma$ is the volume phase fraction.

The VOF method is used for free surface tracking (Hirt \& Nichols, 1981). In the VOF method, the volume phase fraction $\gamma$ indicates the quantity of water per unit volume of each cell (i.e., $0 \leq \gamma \leq 1$ and values 0 and 1 for the regions containing respectively only air or only water). A transport equation is solved for $\gamma$ (Eq. 3). 


$$
\frac{\partial \gamma}{\partial t}+\nabla \cdot(\mathrm{U} \gamma)+\nabla \cdot\left(\mathrm{U}_{r} \gamma(1-\gamma)\right)=0
$$

where $U_{r}$ is an artificial velocity, only active at the interface, which is introduced to limit the smearing of the interface.

The relaxation zone technique of waves2Foam (Jacobsen et al., 2012) is used for waves generation/absorption. At the domain inlet, a boundary condition is defined to introduce waves according to the selected wave theory. Inside the relaxation zones, velocity components and wave surface elevation are imposed by weighing predefined analytical and solved solutions (Eq. 4 and Eq. 5).

$$
\alpha_{R}=1-\frac{\mathrm{e}^{\left(\chi_{R}^{3.5}\right)}-1}{e^{(1)}-1}
$$

where $\Phi$ indicates either $\gamma$ or $U$ and $\chi_{\mathrm{R}}$ is defined to have $\alpha_{\mathrm{R}}=1$ at the end of the relaxation zone.

The $k-\varepsilon$ turbulence model is used, with standard values of the model parameters: $C_{\mu}=0.09$, $C_{1 \varepsilon}=1.44, C_{2 \varepsilon}=1.92$ and $\sigma_{\varepsilon}=1.30$ (Versteeg, 2007). Such a turbulence model has been widely applied to simulate the hydrodynamics and aerodynamics of OWC systems, showing satisfactory performance in terms of validation with laboratory results (e.g. in Lopez et al., 2014, Vizikas et al., 2017, Mendonça et al., 2018).

The PIMPLE algorithm, which is hybrid between the PISO and the SIMPLE algorithm (Jasak, 1996), is used for the coupling of the equations in the pressure-velocity system. For all the simulations in this work, the time step was automatically adjusted in order to maintain a value of the Courant number Co<0.4.

The numerical schemes used for the discretization of time derivatives are second order accurate, blended with a first order Euler scheme to improve stability. A standard finite volume discretization of Gaussian integration is applied to gradient operators, with a central differencing scheme for cell-center to cell-face interpolation. The convection term in momentum equation is discretized with a central difference interpolation scheme. The convection term in the transport equation of the phase fraction $\gamma$ uses the Monotone Upwind Scheme for Scalar Conservation Laws (MUSCL) interpolation scheme. A generalized Geometric-Algebraic Multi-Grid solver (GAMG) is used to solve the discretized system of equations.

\section{NTW domain discretization and sensitivity to mesh resolution}

The computational domain considered for the two-dimensional NWT has a total length and height equal to that of the wave-current flume of the Laboratory of Maritime Engineering of Florence University (www.labima.unifi.it), i.e. respectively $37 \mathrm{~m}$ and $0.8 \mathrm{~m}$. In the NWT, waves are generated and absorbed based by means of the so-called relaxation zone approach, as implemented in the toolbox waves 2 Foam (Jacobsen et al., 2012).

The inlet relaxation zone has a length of $4 \mathrm{~m}$ (corresponding to $1.7-4 \cdot \mathrm{L}$ for the range of tested waves, being $\mathrm{L}$ the wave length) to allow for a proper generation/absorption of the incident wave. The OWC structure is located at the end of the NWT (Fig. 3), therefore a relaxation zone is not used at the outlet (indeed, the quantification of the wave reflection from the structure is one of the aims of this work). No slip boundary conditions are used at the bottom of the NWT and on the OWC side walls, the water surface is set as an atmospheric pressure boundary. Velocity components and water surface elevations at the inlet are defined to introduce regular waves with waves2Foam toolbox.

The mesh is refined in the free surface zone, with a resolution of around 20 cells per wave height $H$ and a maximum cells aspect ratio equal to 2 (Fig. 3). The resulting mesh, of the whole NWT, has a size of approximately 400 ’000 cells. 


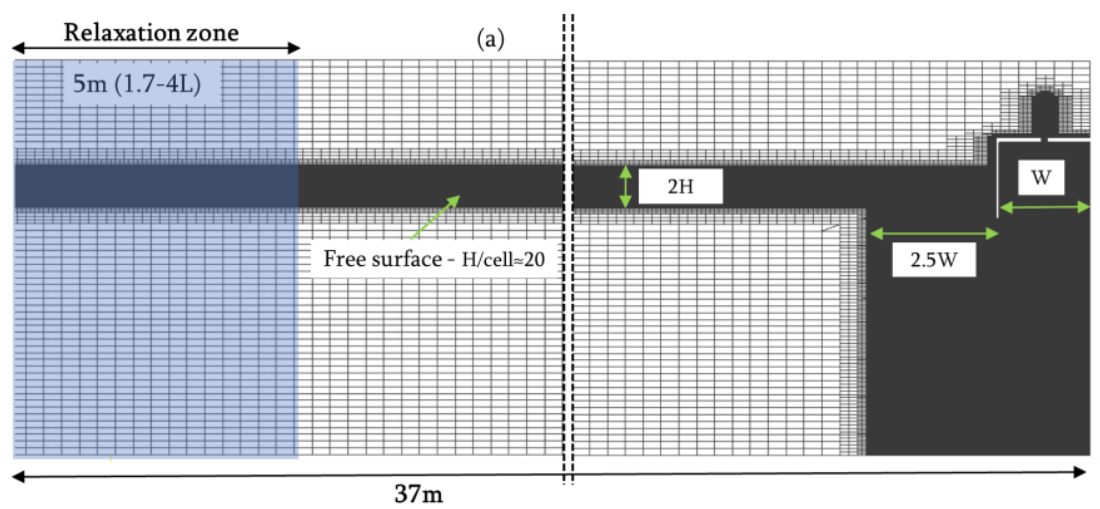

(b)

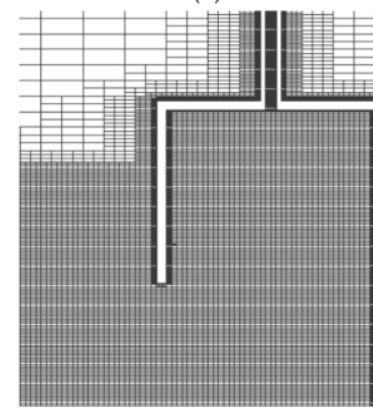

Figure 3. Numerical Wave Tank (NWT) (a), close-up of the computational mesh in the region around the OWC structure (b).

(a)

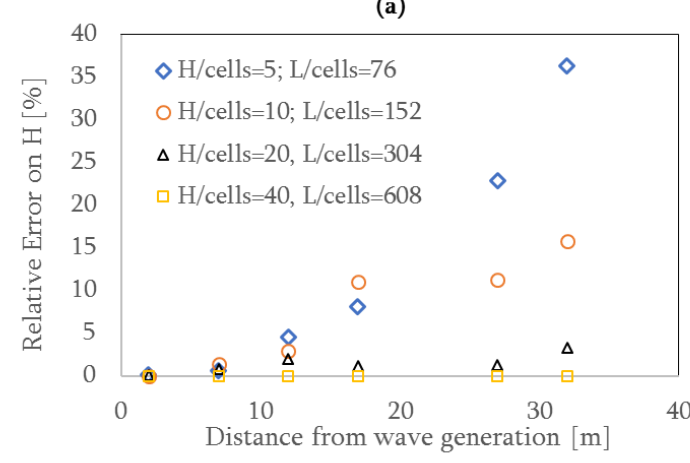

(c)

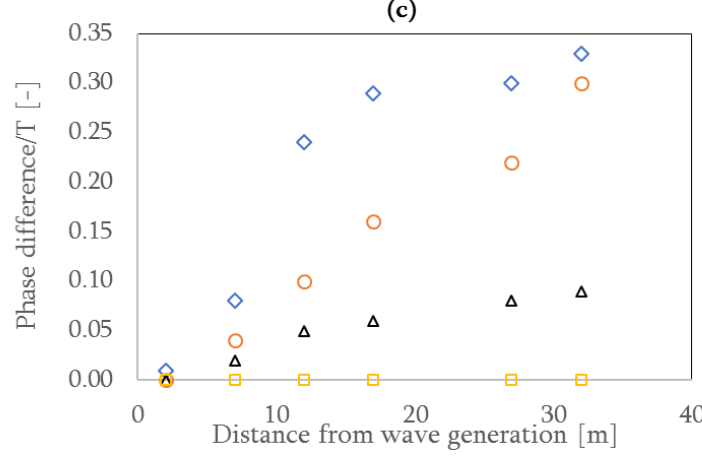

(b)

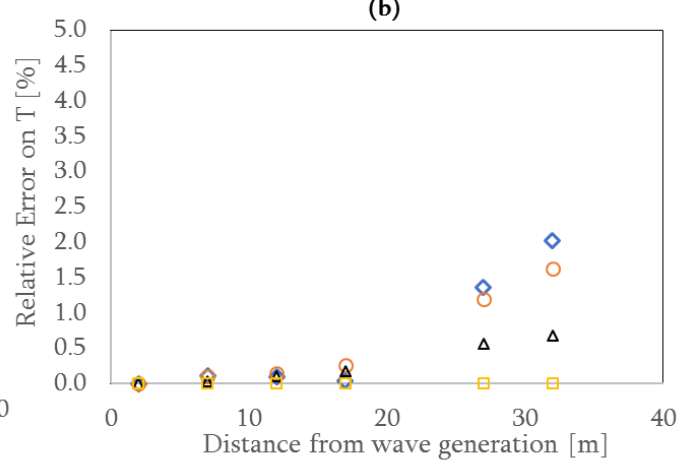

(d)

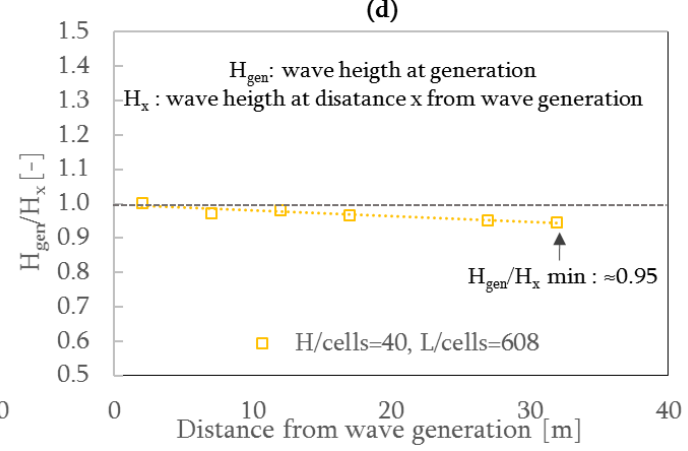

Figure 4. Sensitivity of wave height $H(a)$, wave period $T(b)$ and phase difference (c) to the mesh resolution in the free surface region, for distances from the wave generation varying between $2 \mathrm{~m}$ (corresponding to $2 L$ ) and $32 \mathrm{~m}$ (corresponding to $32 L$ ): results relative to the case with the higher mesh resolution $(H / c e l l s=40$ \& $L /$ cells $=608$ ) and for incident wave with height $H=0.05 \mathrm{~m}$ and period $T=1 \mathrm{~s}$. Ratio of the generated wave height $H_{g e n}$ to the wave height at a distance $x$ from wave generation $H_{x}$ for mesh resolution $H / c e l l s=40 \& L / c e l l s=608$ (d).

The mesh resolution has been chosen based on sensitivity tests aimed at ensuring a substantial mesh independence for the wave propagation along the NWT, at different distance from the wave generation boundary (Fig. 4).

Adopting a resolution higher than 20 cells per wave height, the increase in the model accuracy is negligible: the relative error on $H, T$ and the relative phase difference with respect to the case with resolution $\mathrm{H} /$ cells $=40 \& \mathrm{~L} /$ cells $=608$ are lower than $5 \%, 3 \%$ and $8 \%$ respectively, even for the greater distance considered along the wave propagation direction.

It is worth to note that an increasing phase difference is observed when decreasing the mesh resolution, while the relative error on the wave period $T$ is always negligible (lower than $3 \%$ ). A lower resolution, therefore, causes an improper simulation of the wave kinematics, introducing an error on the wave celerity which accumulates while progressing in time and space. It should be mentioned that aiming to 
simulate the whole extension of the laboratory wave-current flume with the numerical model, a resolution much higher than that needed to only simulate the near-field is required: if the extension of the computational domain was limited to 5-10L (i.e., around $10 \mathrm{~m}$ for the range of wave lengths considered in this work), a resolution of around $H /$ cell=10 would have been enough to ensure a relative error lower than $10 \%$ on the simulated wave height $H$.

Considering the ratio of the generated wave height $H_{\text {gen }}$ to the wave height at a distance $\mathrm{x}$ from wave generation $H_{x}$ for the case with the higher mesh resolution $H /$ cells=40 \& L/cells=608 (Fig. 4,d), a minimum value $H_{g e n} / H_{x}=0.946$ is found. Therefore, the wave damping along the NWT, which might be induced by the RANS turbulence modelling approach adopted (as suggested by Kamath et al., 2015 and Devolder et al., 2017) can be considered negligible for the applications in this study (characterized by values of wave steepness $H / L=0.01-0.04$ ). Worth to note that negligible wave damping along the NWT for relatively low steepness waves, as those considered in this work, is also confirmed in the literature, while higher damping effects could take place for steep waves (as discussed by Devolder et al., 2017 for the $k$ - $\omega$ SST turbulence model).

\section{NTW validation}

The comparison between the numerical model prediction and the results of laboratory-scale tests carried out in the wave-current flume of Florence University shows that the performance of the numerical model in predicting the quantities of interest is quite good (Fig. 5). The relative error between the experiments and the NWT results is lower than $15 \%$ for the pressure $\left(p_{\text {owc }}\right)$ and the water level $\left(\eta_{\text {owc }}\right)$ inside the OWC chamber, which is considered acceptable.

Moreover, considering more specifically the value of the global reflection coefficient $K_{r r}$ (which includes both the contributions of properly reflected and radiated waves) determined by applying the methodology described in Figure 1 ( $1^{\text {st }}$ setp), the results of the numerical model match those obtained with physical modeling quite closely (i.e., the maximum observed relative error on $K_{r r}$ is around $10 \%$ ).
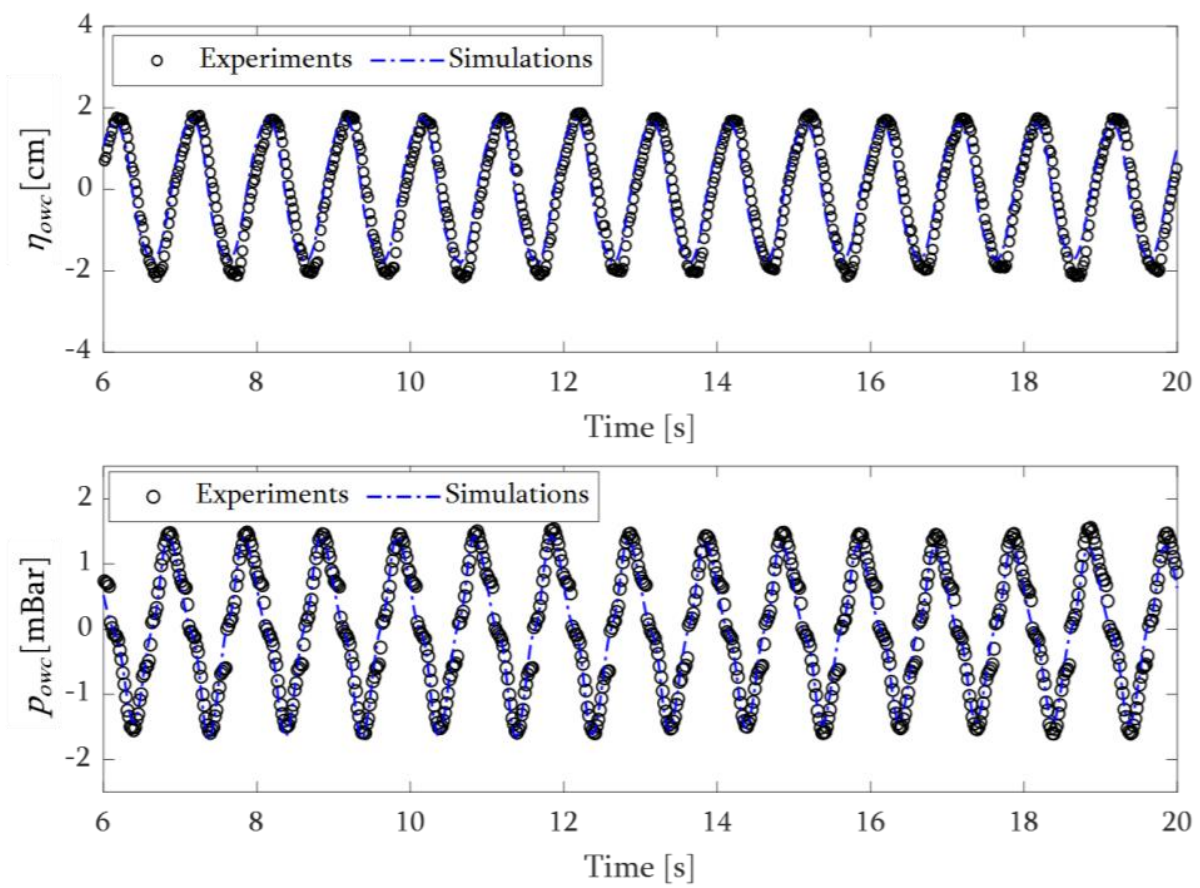

Figure 5. Comparison between laboratory tests and NWT simulations for the pressure $\left(p_{\text {owc }}\right)$ and the water level $\left(\eta_{\text {owc }}\right)$ inside the OWC chamber. Quantities at small scale, for an incident wave with height $H=4 \mathrm{~cm}$ and $T=1 \mathrm{~s}$, and OWC chamber with draught $D=0.09 \mathrm{~m}$, width $W=0.19 \mathrm{~m}$ and top cover orifice diameter $V=0.014 \mathrm{~m}$.

\section{RESULTS AND DISCUSSION}

As aforementioned, the wave field in front of the OWC device results from the interaction of incident, reflected and radiated waves. The resulting agitation is determined by the phase difference 
between the wave components, which dictates whether such components sum up or cancel out (destructive interference).

The total amount of wave energy in front of the OWC is strongly related to the energy absorbed by the device. Indeed, the magnitude of the radiated waves increases as the oscillation of the water column increases, while the reflected component decreases. The phase difference between the wave components is mainly determined by the width of the OWC chamber $W$.

A global reflection coefficient, $K_{r r}$ (Eq. 6), which includes the contribution of both the properly reflected $\left(H_{r e f}\right)$ and the radiated $\left(H_{r a d}\right)$ waves, is defined and analyzed for different OWC structure geometries and incident waves $\left(H_{\text {inc }}\right)$.

The radiated wave is then explicitly measured with the methodology depicted in Figure 1 ( $2^{\text {nd }}$ step), and the interaction between reflected and radiated waves is investigated (Fig. 6).

The radiation coefficient $K_{\text {rad }}$ is estimated as in Eq. 7. The reflected wave is determined as linear superposition of the measured components.

$$
\begin{gathered}
K_{r r}=\frac{\left(H_{r e f}+H_{r a d}\right)}{H_{i n c}}=\frac{H_{r r}}{H_{i n c}} \\
K_{r a d}=\frac{H_{r a d}}{H_{i n c}} \quad K_{r e f}=\frac{H_{r e f}}{H_{i n c}}
\end{gathered}
$$
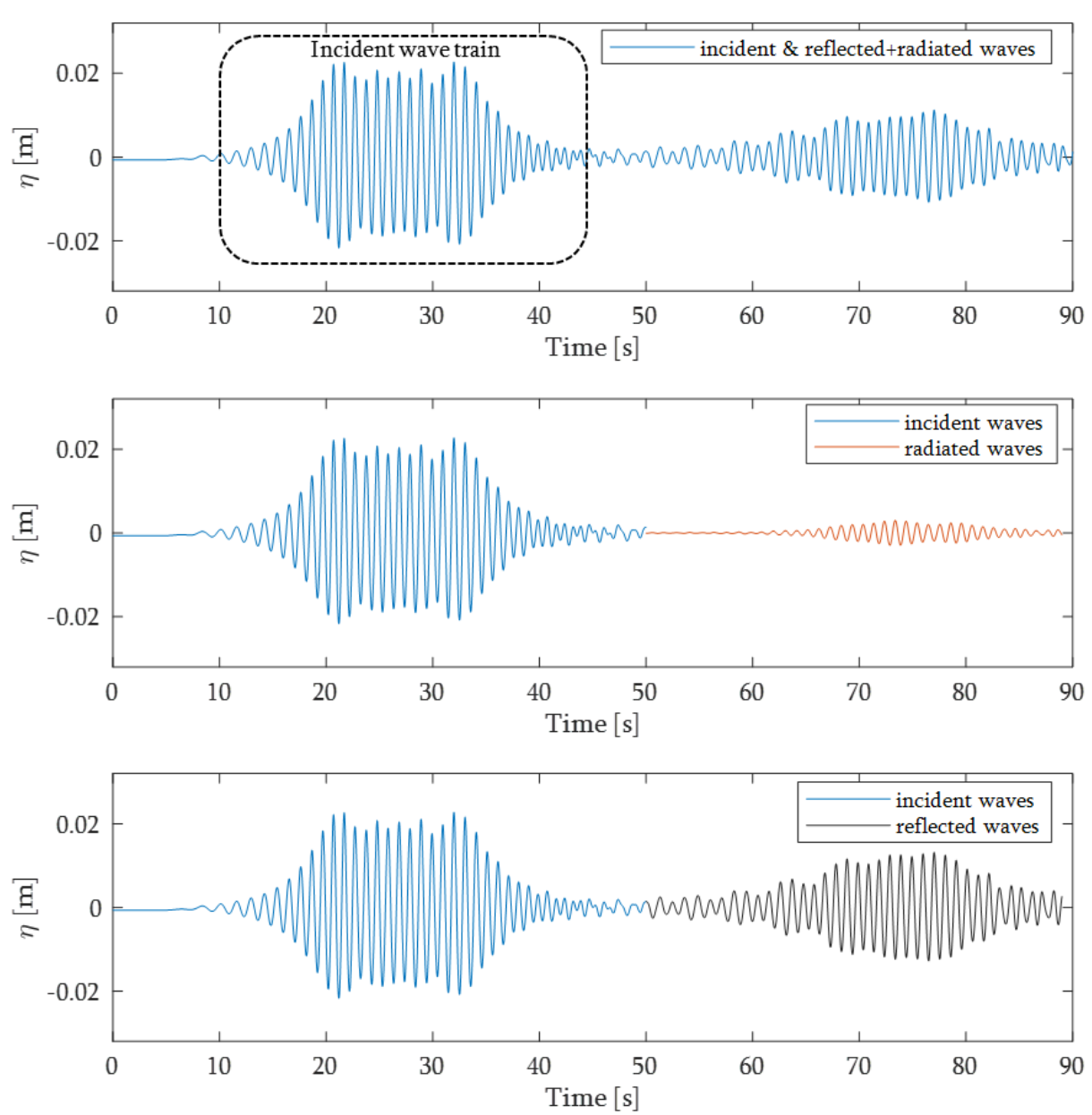

Figure 6. Time series of free surface elevation $\eta$ at $20 \mathrm{~m}(\sim 13 \cdot \mathrm{L})$ from the $\mathrm{OWC}$ structure $(W / L=0.13, V / W=0.050$, $D / H=1.67)$ for an incident wave having $H=4 \mathrm{~cm}$ and $T=1 \mathrm{~s}$. Incident wave train and: reflected plus radiated waves (top panel), radiated waves (central panel), reflected waves (bottom panel). 

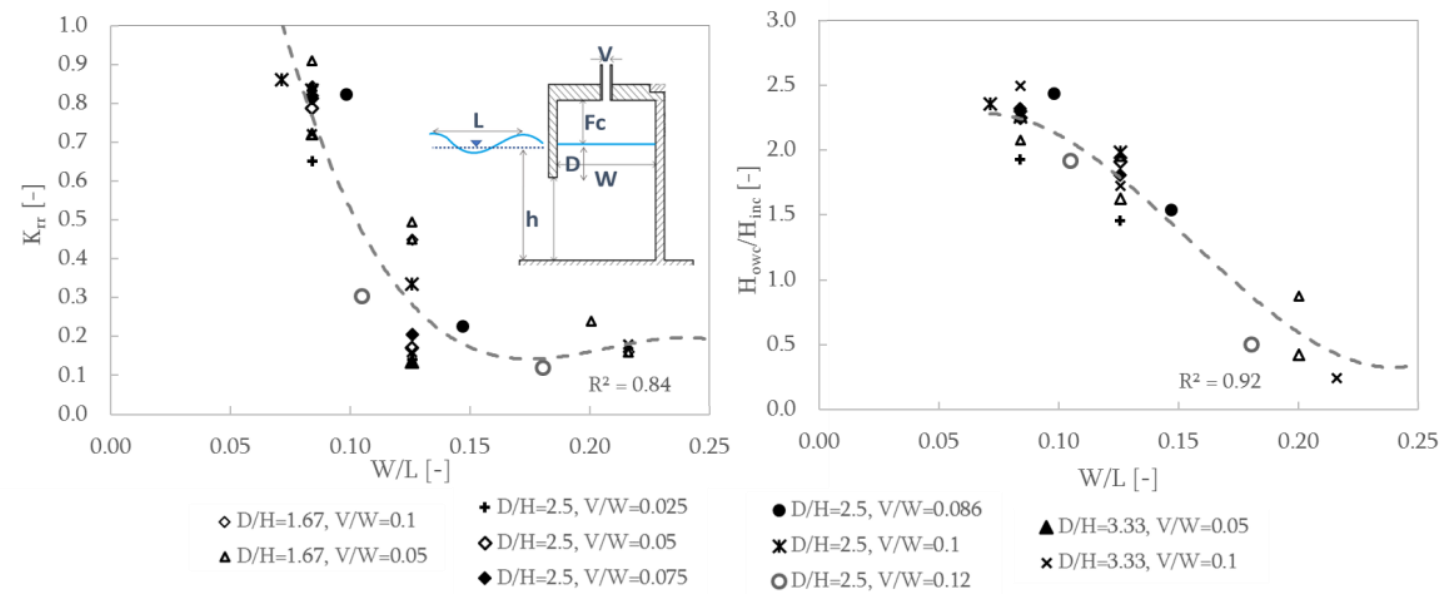

Figure 7. Global reflection coefficient $K_{r r}$ (left) and relative height of the OWC inner water column oscillation relative to the incident wave height, $H_{\text {owd }} / H_{\text {inc }}$ (right) versus $W / L$.

The presence of the OWC embodied into the vertical-wall harbor structure changes the wave field in front of the OWC form a perfect standing wave to a partial standing wave, in which the location of the node slightly varies based on the OWC parameters (mainly the chamber width $W$ ).

Within the geometries tested in this work, the global reflection coefficient $K_{r r}$ shows minimum values of about $0.2-0.15$, for $W / L$ between 0.15 and 0.22 . For $W / L$ lower than 0.1 , remarkably higher values of $K_{r r}(>0.6)$ are found. Valuers of $K_{r r}$ higher than 0.9 are found for $W / L=0.08, D / H=1.67$ and $V / W=0.05$.

The relative chamber width $W / L$ has a paramount impact on $K_{r r}$, i.e. on the over value of the overall wave reflection and radiation from the OWC chamber, and on the way these two components sum up in phase (i.e., destructive or constructive interference).

Other design parameters of the OWC, however, also have a relevant effect on $K_{r r}$, e.g. the relative applied damping $V / W$ and the relative front wall draught $D / H$. Indeed, for fixed value of $W / L=0.13$ and $V / W=0.05, K_{r r}$ ranges between 0.13 and 0.49 (Fig. 7, left): the lower values of $K_{r r}\left(K_{r r}=0.13\right)$ are found for higher values of the relative front wall draught $D / H=3.33$, while decreasing $D / H, K_{r r}$ progressively increases to 0.17 and 0.49 for $D / H=2.5$ and 1.67 respectively.

For the same value of $W / L=0.13$ and a fixed value of $D / H=2.5, K_{r r}$ shows an increasing trend with increasing $V / W$, varying between 0.14 (for $V / W=0.025$ ) and 0.34 (for $V / W=0.1$ ). It is worth to mention, however, that it is expected that further increasing $D / H$ over a threshold value (determined by the mutual effect of all the OWC design parameters on its performance) may result in a larger reflection, due to the increased blockage effect of the front wall of the OWC.

The relative height of the free surface oscillation inside the OWC chamber $\left(H_{\text {owd }} / H_{\text {inc }}\right.$, Fig. 7 , right $)$ is computed based on the value of the free surface level sampled at the center of the OWC chamber. For the geometries tested in this study, it varies between approximately 0.25 (for $W / L=0.22$ ) and 2.5 (for $W / L=0.08$ ), being primarily influenced by the value of the relative chamber width $W / L$, as shown in Figure 7 (right).

\section{The radiated wave field and the radiation coefficient $K_{r a d}$}

The radiated wave amplitude and the resulting radiation coefficient $K_{\text {rad }}$ (Eq. 7), estimated by applying the aforementioned methodology, are determined by the amplitude of the pressure fluctuation inside the OWC chamber, $\triangle P$ Powc. For values of the dimensionless pressure coefficient $\Delta P o w c /\left(2 \cdot \rho \cdot g \cdot H_{\text {inc }}\right)$ varying from around 0.01 to $0.6, K_{\text {rad }}$ varies between around 0.02 to around 0.45 (Fig. 8, left). The rate of increase of $H_{\text {rad }} / H_{\text {inc }}$ with the dimensionless pressure $\Delta P o w c /\left(2 \cdot \rho \cdot g \cdot H_{\text {inc }}\right)$ is also a function of the relative water depth $k h$ (being $k$ the wave number and $h$ the water depth): higher values of $K_{\text {rad }}$ are found for higher values of $k h$ (which correspond to shorter incident wave period, for a fixed value of $h$ ).

Both destructive and constructive interference phenomena are observed in the simulated OWC geometries. The ratio of the radiated wave height to the sum of radiated and reflected wave heights reaches a maximum of $H_{r a d} / H_{r r}=3$ (Fig. 8, right and Fig. 9, left), for the OWC configuration having $W / L=0.13, V / W=0.025$ and $D / H=2.5$. In this condition, the phenomenon of destructive interference 
between the properly reflected and the radiated wave fields is remarkable (Fig. 9, left): the wave components are almost $180^{\circ}$ out of phase, and $H_{r e f}$ is reduced of about $70 \%$ as a consequence of the interaction with the radiated wave. This effect is mainly governed by the chamber width $W / L$, but also the draught $D / H$ and the damping $V / W$ have an influence on the resulting wave height $H_{r r}$. For the same relative width $W / L=0.13$, a lower attenuation of the reflected wave $H_{\text {ref }}$ is found for higher values of the orifice aperture $V / W$ (i.e. lower applied damping) and lower relative draught $D / H$ (Fig. 9, right). For this case, the height of the wave radiated by the OWC is lower, resulting in a reduced impact of the destructive interference on $H_{r r}$.
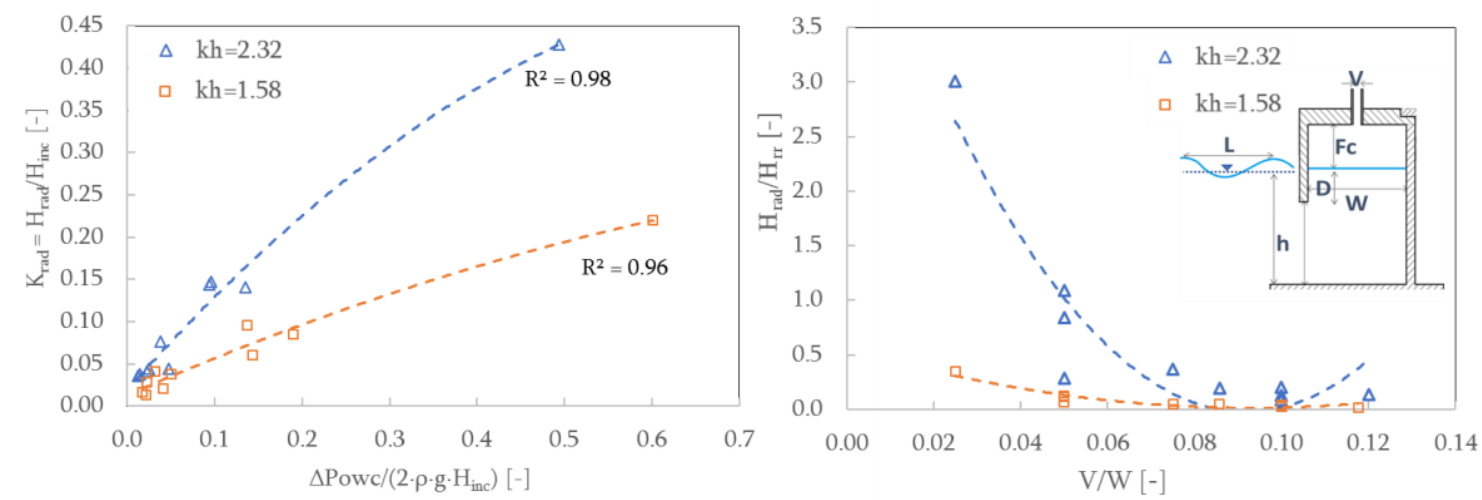

Figure 8. Radiation coefficient $K_{\text {rad }}$ versus dimensionless pressure $\Delta P o w c /\left(2 \cdot \rho \cdot g \cdot H_{\text {inc }}\right)$ (left); radiated wave height relative to the sum of properly reflected and radiated waves $\left(H_{r a d} / H_{r r}\right)$ versus $V / W$ (right). Values for relative water depth $k h=2.32$ and $k h=1.58$.
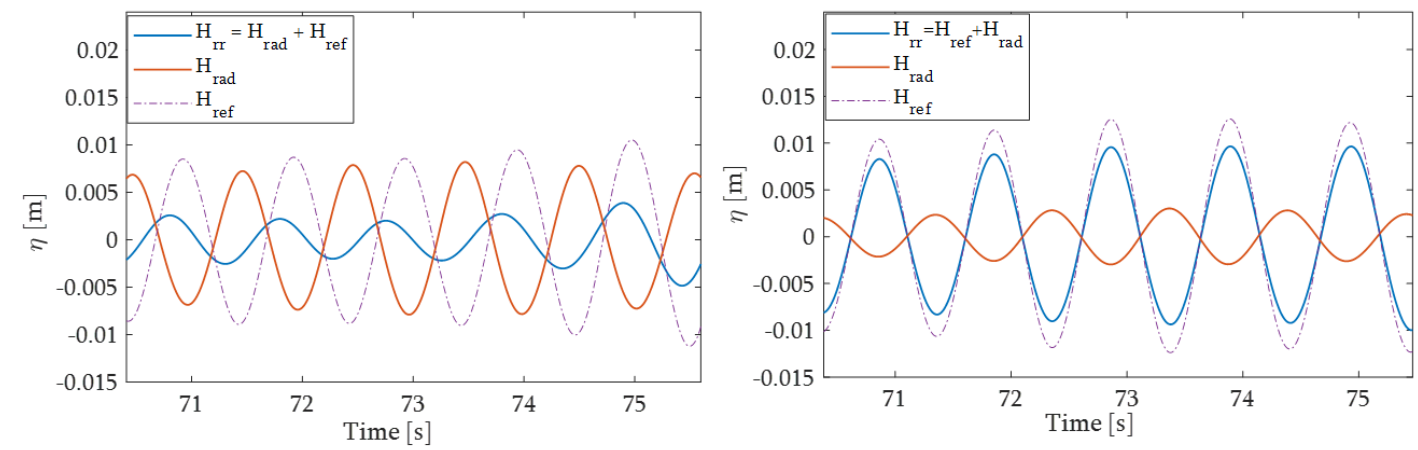

Figure 9. Decomposition of the radiated $\left(H_{\text {rad }}\right)$ and reflected $\left(H_{\text {ref }}\right)$ wave components at $20 \mathrm{~m}(\sim 13 \cdot L)$ from the OWC structure for incident wave $H=4 \mathrm{~cm}$ and $T=1 \mathrm{~s}$ and relative water depth $k h=2.31$. Results for $O W C$ geometries $W / L=0.13, V / W=0.025, D / H=2.5$ (left) and $W / L=0.13, V / W=0.050, D / H=1.67$ (right).

\section{The OWC capture width ratio}

As an index of the capability of the OWC structure to extract part of the incident wave energy to be later converted into a usable form, the Capture Width Ratio (CWR) of the device is calculated as the ratio of the period-averaged pneumatic power extracted by the OWC $\left(P_{o w c}\right)$ to the period-averaged incident wave power $\left(P_{\text {wave }}\right)$.

$$
\begin{gathered}
C W R=\frac{P_{o w c}}{P_{\text {wave }}} \\
P_{o w c}=\frac{1}{T_{\text {test }}} \int_{0}^{\text {Ttest }} p_{\text {owc }}(t) \frac{d \eta_{\text {owc }}}{d t} \cdot W d t[\mathrm{~W} / \mathrm{m}]
\end{gathered}
$$

where $p_{\text {owc }}$ and $\eta_{\text {owc }}$ are the instantaneous values of air pressure and water level inside the OWC chamber, $T_{\text {test }}$ is the duration of the analysis. For regular incident waves, the period-averaged incident wave power per unit width $[\mathrm{W} / \mathrm{m}]$, for a generic water depth $h$, can be computed as 


$$
P_{\text {wave }}=\frac{1}{16} \rho g H^{2} \frac{\omega}{k}\left(1+\frac{2 k h}{\sinh (2 k h)}\right)
$$

where $\rho$ is the water density, $H$ is the regular wave height, $\omega$ is the wave frequency and $k$ is the wave number.

For the OWC geometries studied in this work, increasing values of CWR are found when decreasing the relative orifice aperture $V / W$, i.e. when increasing the level of damping applied to the OWC chamber (Fig. 10). A maximum value of CWR of around 0.55 is found in this study. Such a maximum value is obtained for $W / L=0.13, V / W=0.025$ and $D / H=2.5$, with a corresponding value of the global reflection coefficient $K_{r r}$ of about 0.15 .

It is expected that greater values of CWR can be obtained by adapting the geometry parameters of the OWC (as discussed in Simonetti et al., 2017) while maintain relatively satisfactory performance of the device in terms of reflection reduction.

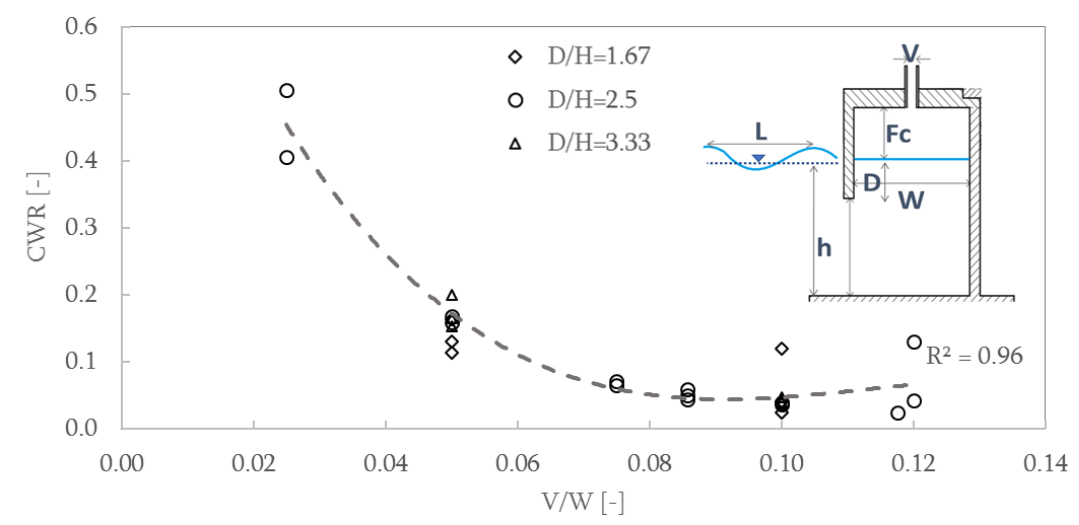

Figure 10. Capture Width Ratio (CWR) of the OWC device versus relative orifice aperture V/W.

\section{CONCLUSIONS AND OUTLOOKS}

The possibility of using OWC devices to reduce the agitation in front of vertical wall harbor structures is investigated, with a particular focus on the global wave reflection (i.e., the sum of properly reflected and radiated waves) and on the interaction among reflected and radiated fields.

Minimum values of the global reflection coefficient $K_{r r}$ of around 0.15 are found, meaning that the OWC device could be effectively used to reduce wave reflection at vertical wall harbor structures: the minimum $K_{r r}$ is comparable to that obtained for slotted-barrier-type wave absorber by Zhu and Chwang, 2001 (i.e. around 0.2) and to that achievable with partially perforated Jarlan type structures (Huang et al., 2011). Moreover, compared to more consolidated wave absorbers structures, the use of OWC devices would allow to harvest the wave energy into a usable form, rather than just dissipating it. Within the tested conditions, when $K_{r r}$ is at its minimum, the OWC has a value of the Capture Width Ratio of around 0.55. By optimizing the geometry parameters of the device, the wave energy extraction performance could further be improved.

The wave radiated by the OWC structure is determined by the amplitude of the pressure fluctuation inside the OWC chamber, $\triangle P o w c$, with increasing radiated wave heights for increasing $\triangle P o w c$. The value of $\triangle P O w c$ is, in turn, fundamentally determined by the damping applied by the orifice that connects the OWC interior with the atmosphere. The interaction of the radiated and the reflected wave fields, i.e. the way the phase of these components sums up to determine constructive or destructive interference, is instead mainly related to other structural parameters, e.g. the OWC chamber width in wave propagation direction. For the OWC geometries tested in this work, the radiated wave was found to be capable of attenuating the wave reflected by the structure up to around $70 \%$, through the phenomenon of destructive interference.

This work focusses only on the analysis of the interaction of reflected and radiated wave fields in front of on OWC device integrated into a harbor breakwater. Future developments of the present work should include extended study of the wave field in proximity of the OWC structure, comprising therefore an in depth analysis of the interaction between the incident and the reflected plus radiated wave fields, possibly providing an index to characterize the water surface agitation inside the harbor as a function of 
the OWC design parameters and wave conditions. The number of tested geometries and wave conditions should also be extended in future studies, aimed, in particular, to assess the capability of the device to attenuate longer waves.

In this work, $H_{\text {owc }}$ is sampled at the center of the OWC chamber, i.e. a flat piston-type heaving motion of the OWC inner surface is assumed. It has to be underlined that relevant spatial variations of the surface elevation inside the OWC chamber may take place, particularly for relatively short waves. These spatial variations in the free surface may induce errors in the estimation of CWR, as pointed out in (He and Huang, 2016b). Future extensions of these work should therefore also address such aspect.

\section{REFERENCES}

Altomare, C., X. Gironella. 2014. An experimental study on scale effects in wave reflection of lowreflective quay walls with internal rubble mound for regular and random waves. Coastal Engineering, 90, pp. 51-63.

Ciocan, C.R., Taveira-Pinto, F., das Neves, L., and P. Rosa-Santos. 2017. Experimental study of the hydraulic efficiency of a novel perforated-wall caisson concept, the LOWREB, Coastal Engineering, 126, pp. 69-80.

Devolder, B., Rauwoens, P., Troch, P. 2017. Application of a buoyancy-modified k- $\omega$ SST turbulence model to simulate wave run-up around a monopile subjected to regular waves using OpenFOAM®, Coastal Engineering, 125, pp: 81-94.

Elhanafi, A., Fleming, A., Macfarlane, G., and Z. Leong. 2017. Underwater geometrical impact on the hydrodynamic performance of an offshore oscillating water column wave energy converter, Renewable Energy, 105, 209-231.

Falcao, A.F.O, and J.C.C Henriques. 2016. Oscillating-water-column wave energy converters and air turbines: A review. Renewable Energies, 85, 1391-1424.

Falnes, J. 2002. Ocean Waves and Oscillating Systems: Linear Interactions Including Wave-Energy Extraction, Cambridge University Press, ISBN 10: 0521782112.

Garrido, J., Ponce, D., Berruguete, A., Martinez, S., Manuel, J., Fort, L., Yagüe, D., Gonzalez-Escriva, J.A., and J.R. Medina. 2010. Study of reflection of new low-reflectivity quay wall caisson. Proceedings of 32nd International Conference on Coastal Engineering, ASCE, pp. 466-480.

Goda, Y., Suzuki, T., and H. Okuzono. 1976. Estimation of incident and reflected waves in random wave experiments. Coastal Engineering Proceedings 1 (15). http://dx.doi.org/10.9753/icce.v15.

Gonzalez-Escriva, J., and J. Medina. 2012. Antireflective vertical structure extended for attenuation of low frequency waves. Coastal Engineering Proceedings, 1(33).

He, F., Li, M., and Z. Huang. 2016a. An Experimental Study of Pile-Supported OWC-Type Breakwaters: Energy Extraction and Vortex-Induced Energy Loss, Energies, 9, 540-555.

He, F., and Z. Huang. 2016b. Using an oscillating water column structure to reduce wave reflection from a vertical wall. Journal of Waterway, Port, Coastal and Ocean Engineering, 142(2).

Hirt C. and B. Nichols. 1981. Volume of fluid (VOF) method for the dynamics of free boundaries. $J$. Comput. Phys. 39, pp: 201-225.

Huang, Z., Li, Y., and Y. Liu. 2011. Hydraulic performance and wave loadings of perforated/slotted coastal structures: A review. Ocean Engineering, 38, pp. 1031-1053.

Jacobsen N.G., Fuhrman D.R., and J. Fredsøe. 2012. A wave generation toolbox for the open-source CFD library : Open-Foam. Int. J. Num. Methods Fluids, 70, pp: 1073-88.

Jarlan, G.E. 1961. A perforated vertical wall breakwater. The Dock and Harbor Authority, 41, n. 486 pp. 394-398.

Jasak, H. 1996. Error analysis and estimation for the finite volume method with applications to fluid flows. PhD thesis, Imperial College of Science, Technology and Medicine, London, UK.

Kamath, A., Chella, M.A., Bihs, H., and Arntsen, Ø.A. 2015. CFD investigations of wave interaction with a pair of large tandem cylinders, Ocean Engineering, 108, pp:738-748.

Liu, C.R., and N. Geng. 2012. Anti-reflection characteristics of oscillating water column wave energy device, Journal of Hunan University Natural Sciences, 39(2), pp. 35-40.

Lopez, I., Pereiras, B., Castro, F., and G. Iglesias. 2014. Optimization of turbine-induced damping for an OWC wave energy converter using a RANS-VOF numerical model, Applied Energy 127 pp: 105114.

Lopez, I., Rosa-Santos, P., Moreira, C., and F. Taveira-Pinto. 2018. RANS-VOF modelling of the hydraulic performance of the LOEREB caisson, Ocean Engineering, 140, 161-174. 
Mansard, E.P.D., and E.R. Funke. 1980. The measurement of incident and reflected spectra using a least square method. Proc. 17th Int. Conference on Coastal Engineering, ASCE, Sydney.

Mendonça, A., Dias, J., Didier, E., Fortes, C.J.E.M., Neves, M.G., Reis, M.T., Conde, J.M.P., Poseiro, P., and P.R.F. Teixeira. 2018. An integrated tool for modelling oscillating water column (OWC) wave energy converters (WEC) in vertical breakwaters, Journal of Hydro-environment Research, 19, pp: 198-213

Morris-Thomas, M.T., Irvin, R.J., and K.P. Thiagarajan. 2007. An investigation into the hydrodynamic efficiency of an oscillating water column, J. Offshore Mech. Arct. Eng., 129(4), pp: 273-278.

Neelamani, S., Al-Salem, K., and A. Taqi. 2017. Experimental investigation on wave reflection characteristics of slotted vertical barriers with an impermeable back wall in random wave fields, Journal of Waterway, Port, Coastal and Ocean Engineering, 143 (4).

Oumeraci, H. 2010. Nonconventional wave damping structures. In: Kim, Y.C. (Ed.), Handbook of Coastal and Ocean Engineering. World Scientific.

Sarmento, A., and A. Falcão. 1985. Wave generation by an oscillating surface-pressure and its application in wave-energy extraction. Journal of Fluid Mechanics, 150, 467-485.

Scarpetta, F., Gurnari, L., Torresi, M., Filanioti, P., and S. Camporeale. 2017. A CFD simulation of a full-scale U-OWC Breakwater, Proceedings of the 12th European Wave and Tidal Energy Conference, Cork, Ireland.

Simonetti, I., Cappietti, L., ElSafti, H., and H. Oumeraci. 2017. Optimization of the geometry and the turbine induced damping for fixed detached and asymmetric OWC devices: A numerical study, Energy, 139, pp: 1197-1209.

Simonetti, I., Crema, I., Cappietti, L., El Safti, H., and H. Oumeraci. 2016. Site-specific optimization of an OWC wave energy converter in a Mediterranean area. Progress in Renewable Energies Offshore, Guedes Soares (Ed.). Taylor \& Francis Group, London, pp. 343-350.

Theocharis, I., Anastasaki, E.N., Moutzouris, C.I., and T. Giantsi. 2011. A new wave absorbing quaywall for wave height reduction in a harbor basin, Ocean Engineering, 38, 1967-1978

Versteeg, H.K., and W. Malalasekera. 2007. An Introduction to Computational Fluid Dynamics - The Finite Volume Method, Second ed., Pearson Education Limited. Glasgow, UK, 503 pp.

Vyzikas, T., Deshoulières, S., Giroux, O., Barton, M., and D. Greaves. 2017. Numerical study of fixed Oscillating Water Column with RANS-type two-phase CFD model, Renewable Energy, Volume 102, Part B, pp: 294-305, ISSN 0960-1481, https://doi.org/10.1016/j.renene.2016.10.044.

Zhu, S., and A.T. Chwang. 2001. Investigation on the reflection behavior of slotted seawall. Coastal Engineering, 43(2), 93-104. 